\title{
Ultrastructural Study of the Aphanius dispar (Rüppell 1828) (Pisces: Cyprinodontidae) liver
}

\author{
Taher A. Ba-Omar. M. Al-Amri, R. Victor \\ Biology Department, College of Science, Sultan Qaboos University, P.O.Box 36, \\ Muscat, PC 123, OMAN \\ taher@squ.edu.om
}

Aphanius A. dispar (Rüppell 1828), is widely distributed cyprinodont fish in fresh and brackish waters of the Middle East. It is capable of coping with a variety of difficult environmental conditions. It can adapt to various ranges of habitats that differ in water chemistry, temperature and stream flow. It can live in a wide range of salinity including marine water (Haas, 1982).

The fish were collected and transported into the Biology Department, Sultan Qaboos University, OMAN. The fish were killed by placing them in ice for few minutes. They were dissected out for their liver. The livers were fixed in Karnovsky buffered with sodium cacodylate to a $\mathrm{pH}$ of 7.4 for four hours and then cut into small pieces. The tissue were washed in cacodylate buffer and then post-fixed in $1 \%$ aqueous solution of osmium tetroxide for 1 hour and dehydrated in a series of alcohol before embedding in Agar 100 resin. Semi and ultra-thin sections were cut using Reichert ultramicrotome. The semi-thin sections were stained with toluidine blue and the sections were examined using a Nikon 104 light microscope. The ultra-thin sections were stained with uranyl acetate and post-stained in lead citrate. They were examined under JEOL JEM -1230 TEM.

The liver is composed of a continuous compact of hepatocytes with connective tissue enclosing the bile duct and blood vessels. The hepatocytes are polyhedral in shape and have rounded nuclei (Fig 1 and 2). They contain large amount of glycogen and few mitochondria. Some mitochondria were seen in association with endoplasmic reticulum (Fig. 2)

\section{Reference}

Haas, R. 1982. Notes on the ecology of Aphanius dispar in the Sultanate of Oman. Freshwater Biology. 12: 89-95. 


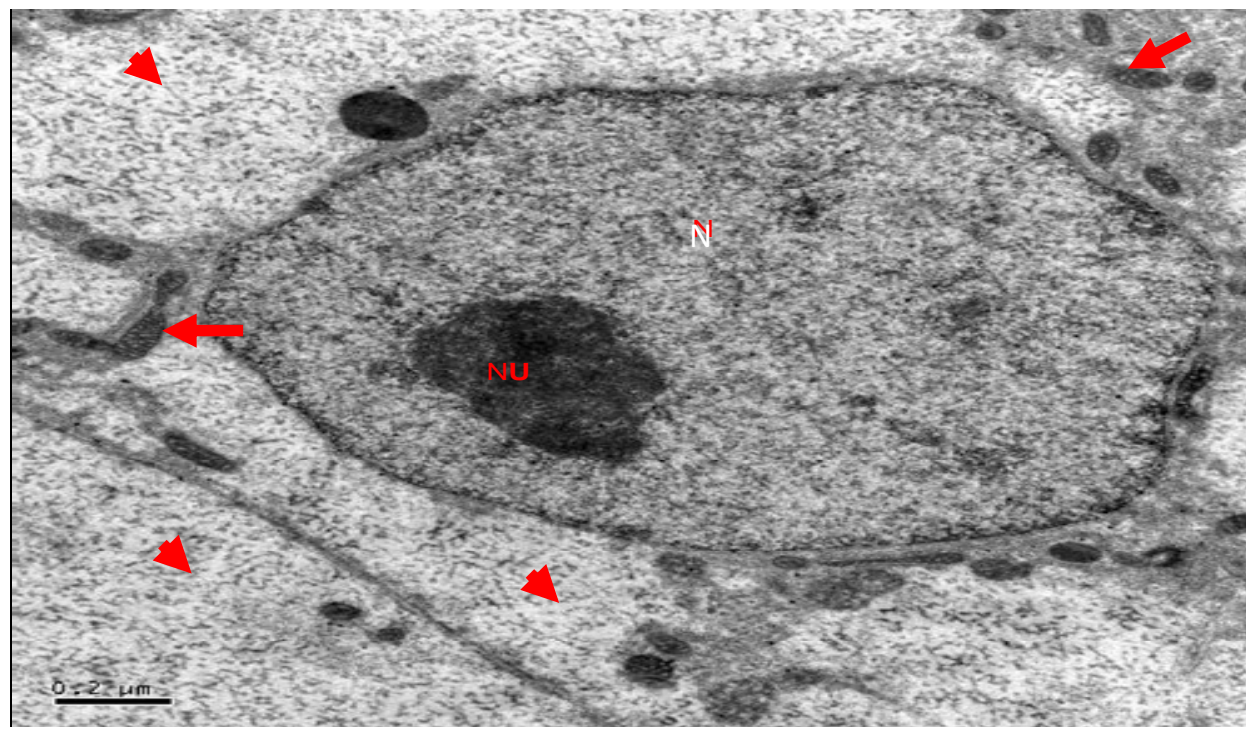

Figure 1: Transmission electron micrograph of hepatocytes of the A. dispar. Nucleus of (N) Mitochondria (arrows) and glycogen dispersed throughout the cytoplasm (arrowheads). . Scale bar $=0.5 \mu \mathrm{m}$

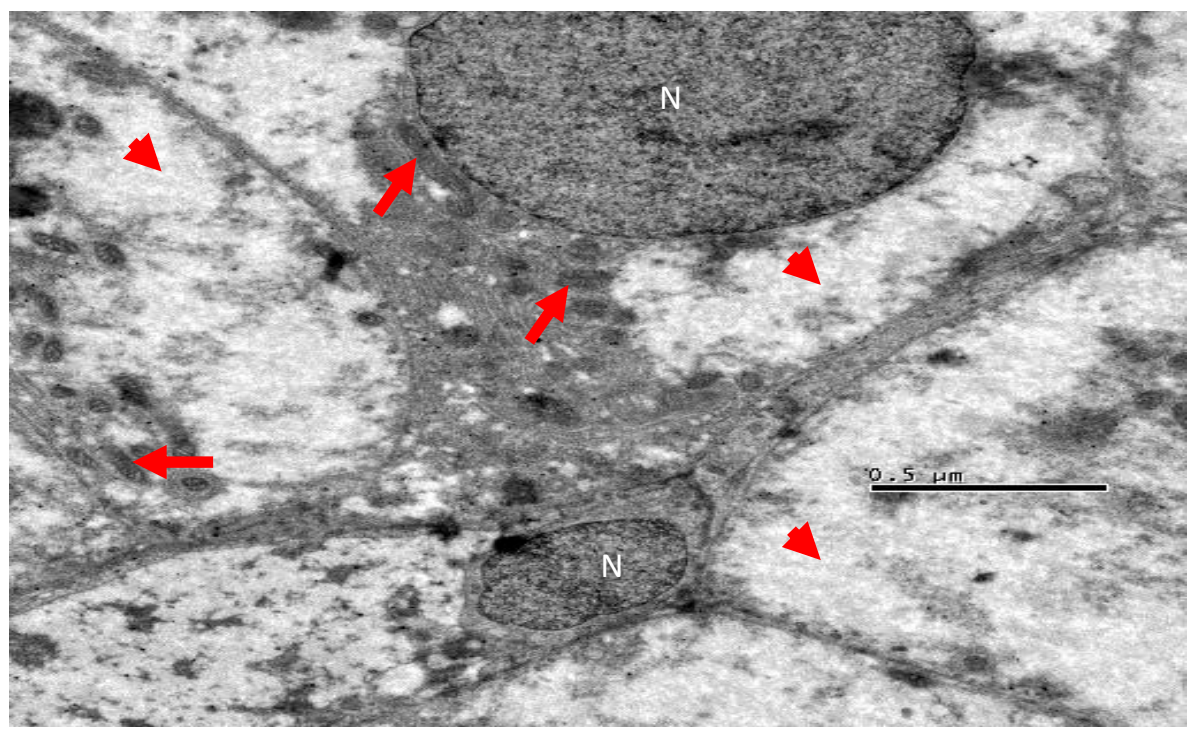

Figure 2: Transmission electron micrographs of hepatocytes of the $A$. dispar. The large centrally located nucleus $(\mathrm{N})$. Mitochondria (arrows) associated with ERcisternae and dispersed throughout the cytoplasm. Glycogen dispersed throughout the cytoplasm (arrowheads). Scale bar $=0.5 \mu \mathrm{m}$ 